\title{
Attitudes of life science majors towards computational modeling in introductory physics
}

\author{
Brandon R. Lunk ${ }^{1,2}$ and Robert J. Beichner ${ }^{3}$ \\ ${ }^{I}$ Department of Physics, Texas State University, Comanche Dr., San Marcos, TX 78666 \\ ${ }^{2}$ Department of Physics, Elon University, 100 Campus Drive, Elon, NC 27244 \\ ${ }^{3}$ Department of Physics, North Carolina State University, 2401 Stinson Drive, Raleigh, NC 27695
}

\begin{abstract}
Biological and health care majors comprise one of the largest populations of students enrolled in physics courses each year. Because of this, there is a growing interest within the physics and biology communities to restructure the introductory physics courses for life science majors to better support the needs of these students. In this context, computational modeling could prove to be an accessible and compelling tool for exploring biologically and medically relevant phenomena within in the physics course. As a first step, we conducted an exploratory study to help us learn about life-science majors' attitudes towards programming. Our observations suggest that while these students held an apprehension towards programming, they appeared to be more receptive towards spreadsheets, suggesting that this tool could provide an accessible way to scaffold more rigorous computational modeling tasks in the classroom.
\end{abstract}

\section{INTRODUCTION}

Biological and health care majors comprise one of the largest populations of students enrolled in physics courses each year. Despite there being deep connections between physics and biological systems, the introductory physics courses for life-science majors (IPLS) are typically treated as "calculus-light" versions of the major-track introductory sequence [1]. Consequently, this marginalizes these courses from the perspective of the life-science communities. In response, there is a growing interest within the physics and biology communities to restructure IPLS courses to better support the needs of the life-science majors [2].

Computational modeling presents itself as a compelling tool in this context. By placing an emphasis on modeling [3] and design [4], while also deemphasizing prohibitively difficult mathematics and including elements of visualization, computational modeling could prove to be an exciting and tractable tool for IPLS students to explore biologically and medically relevant phenomena, such as random motion, viscous friction, and diffusion. Much of the research that has gone into making computational modeling an instructionally viable enterprise, however, has focussed on its use in the major-track $[3,5,6]$ or K-12 science courses [4] rather than in IPLS courses. This suggests an important thread of research to be done.

One prominent consideration is life-science majors' receptiveness to computational modeling in the classroom. As a first step towards understanding this issue, we discuss the results of an exploratory study which we designed to probe lifescience majors' attitudes and expectations towards programming in their college experiences, in their projected professional fields, and in general. Because these issues have not been previously explored, our goal is to help inform the direction of future research. In particular, the results of this study suggest that spreadsheets might provide an accessible entryway to iterative methods and computational modeling for IPLS students and we suggest this as a focus for further research and curricular design.

\section{BACKGROUND}

\section{A. Computational Modeling in Intro. Physics}

While computational modeling in physics has long enjoyed a status on par with theory and experiment, the past few decades have seen the development of programming environments and processor speeds that can enable students to focus on the practice of modeling without being overburdened by esoteric programming details [e.g., 5]. Ongoing efforts at integrating computational modeling into the physics classroom have provided insight into both the logistics of implementation $[3,5]$ as well as details of student reasoning $[4,6,7]$. Again, however, these studies focus on the major-track and K12 courses. Furthermore, most of these efforts emphasize programming languages like VPython; While rudimentary programming can be done through spreadsheet programs like Excel, such software is typically only used in the classroom for data collection and analysis. A few recent efforts have utilized computation (including spreadsheets) in modeling biologically relevant systems [e.g., 8], but the focus of these has not been IPLS courses.

\section{B. Classroom Attitudes}

Student affect within the classroom has been shown to be linked to many factors, including motivation and performance [9]. For this reason, it is important to understand such emotional factors and how they might interact with the use of novel pedagogical tools. Indeed, recent efforts have used programming as the context through which to explore students' sense of identity and self-efficacy [10]. Although this study focused on K-12 students, it provides us with a point of reference for exploring student attitudes towards programming in the general-level physics courses. 


\section{STUDY DESIGN AND METHODOLOGY}

\section{A. Experimental Setup}

We drew data for this study from five clinical interviews that the first author conducted with students enrolled in an introductory, general-level mechanics course at North Carolina State University during the 2014 Fall semester. This course aligned with traditional general-level courses and featured the use of spreadsheets during the accompanying labs for the purposes of data analysis but not for computational modeling. The participants all responded to a general recruitment announcement that was given in all sections of a single instructor's course. These participants had diverse backgrounds, but were all interested in pursuing careers in the biological and health-care fields.

Each interview had two parts. The first was an open-ended, conversational interview which we designed to engage participants on their expectations and attitudes towards science, physics, and computation. Following existing methodologies $[10,11]$, we developed seed questions (e.g., "What do you think of when you think of programming?") with the expectation that followup questions would be generated in situ. We deliberately left the interpretation of these questions to our participants; while it is possible that specifying a particular use for these tools might have produced different responses, our goal was to learn about our participants' perceptions of and receptiveness towards these tools.

For the second part, we had our participants read through a computational model of two-body planetary motion written in VPython and, following the methodology of Weatherford [6], predict the outcome of running the program. We designed this second part to gauge our participants' baseline comprehension however we leave the analysis of this for future work as it is beyond the scope of our present research goals. During the open-ended interview, some participants expressed attitudes toward having to interact with this computational model and we include these reactions in the present analysis.

\section{B. Data Analysis}

After transcribing our participants' interviews and assigning each a pseudonym, we conducted an initial read-through to develop a general sense of each interview and to determine what aspects to analyze. For this paper, we choose restrict our analysis to only those episodes in which computation was germane to the conversation. We eventually segmented the transcripts into individual statements and coded these along four dimensions: conversational topic (spreadsheets or programming, which we take to include coding HTML); participants' attitude (positive or negative); participants' sense of self-efficacy (positive or negative); and the participants' sense for how useful the conversational subject (e.g. spreadsheets) might be. Segments could be coded along multiple dimensions. We should emphasize that these codes are only meant to capture a broad sense for our participants' affective stances.

We tested the reliability of our codes through multiple
TABLE I: Percent of each participant's total statements in which they expressed a positive or negative affective stance towards programming $(\mathrm{P})$ and towards spreadsheets (S). Percentages should be read as "code count/total number of segments".

\begin{tabular}{|c|c|c|c|c|c|c|c|}
\cline { 2 - 8 } \multicolumn{2}{c|}{} & \multicolumn{2}{c|}{ Self-Efficacy } & \multicolumn{2}{c|}{ Attitude } & \multicolumn{2}{c|}{ Useful } \\
\multicolumn{2}{c|}{} & $\mathrm{P}$ & $\mathrm{S}$ & $\mathrm{P}$ & $\mathrm{S}$ & $\mathrm{P}$ & $\mathrm{S}$ \\
\hline \multirow{2}{*}{ Linda } & + & $0 \%$ & $16.7 \%$ & $16.7 \%$ & $8.3 \%$ & $0 \%$ & $8.3 \%$ \\
& - & $16.7 \%$ & $8.3 \%$ & $16.7 \%$ & $16.6 \%$ & $0 \%$ & $8.3 \%$ \\
\hline \multirow{2}{*}{ Reneé } & + & $18.8 \%$ & $6.3 \%$ & $18.8 \%$ & $6.3 \%$ & $12.5 \%$ & $6.3 \%$ \\
& - & $6.3 \%$ & $6.3 \%$ & $6.3 \%$ & $0 \%$ & $0 \%$ & $0 \%$ \\
\hline \multirow{2}{*}{ Sandra } & + & $0 \%$ & $0 \%$ & $0 \%$ & $11.1 \%$ & $0 \%$ & $22.2 \%$ \\
& - & $5.6 \%$ & $5.6 \%$ & $0 \%$ & $0 \%$ & $0 \%$ & $16.7 \%$ \\
\hline \multirow{2}{*}{ Trudy } & + & $0 \%$ & $10 \%$ & $0 \%$ & $15 \%$ & $10 \%$ & $25 \%$ \\
& - & $20 \%$ & $0 \%$ & $5 \%$ & $5 \%$ & $0 \%$ & $0 \%$ \\
\hline \multirow{2}{*}{ Wendy } & + & $7.7 \%$ & $15.4 \%$ & $7.7 \%$ & $23.1 \%$ & $0 \%$ & $30.8 \%$ \\
& - & $15.4 \%$ & $7.7 \%$ & $7.7 \%$ & $0 \%$ & $0 \%$ & $0 \%$ \\
\hline
\end{tabular}

rounds of inter-rater reliability in order to refine our analysis and ensure that it represents a meaningful picture of our participants' responses. We ultimately achieved reliability, yielding Cohen's Kappa values [12] of agreement for topic $(\kappa=0.84)$, attitude $(\kappa=0.83)$, self-efficacy $(\kappa=0.81)$, and sense of usefulness $(\kappa=0.80)$.

\section{RESULTS}

While our principle analysis is qualitative in nature [13], a quantitative description was helpful in characterizing our participants' responses (see Table I; we report percentages to normalize code counts for interview length): Trudy and Wendy held mostly positive affective stances towards spreadsheets while responding negatively to programming; Linda expressed mixed feelings, but had a higher self-efficacy towards spreadsheets (as did Trudy and Wendy); Sandra provided little information about her attitudes beyond expressing a lack of experience; and Reneé, had experience programming and expressed mostly positive stances to both programming and spreadsheets. Everyone except Linda expressed the sense that spreadsheets were more useful than not but only Reneé and Trudy saw programming as a useful tool, though Trudy was mostly speculating about her professors' practices.

We should emphasize that our use of a quantitative analysis here is meant to support our qualitative characterization of the interviews and should not be taken as the basis for a statistical model of student perceptions. (Unsurprisingly, we found no statistical correlation between our participants' combined affective stance and the topic to which they were referringa result that reflects the variation within a small number of responses.)

\section{A. A Case Study: Wendy Expresses Two Views}

To provide a more nuanced picture of our participants' outlooks towards both programming and spreadsheets, we turn to discuss the qualitative aspects of their responses in more de- 
tail. We start with a discussion of Wendy's interview. Wendy was quite expressive and provided an interesting nuance to her responses. We begin with her stance towards programming ${ }^{1}$ :

Interviewer: What do you think of when you think of programming?

Wendy: Programming? ... I'm absolutely terrible with computers. When I think of programming, I kind of think just a lot of numbers and letters and brackets and all kinds of weird languages that I do not understand...

Wendy starts by expressing a lack of confidence in her abilities with computers. She underscores this by expressing the sense that program code is incomprehensible, referring to it as being comprised of "numbers and letters and brackets and all kinds of weird languages." Interestingly, Wendy revealed that she speaks from experience, having once attempted to learn HTML. She expressed mixed emotions about her experience:

Wendy: When I was younger... I tried to teach myself HTML and that's the extent of my programming experience. Everything else is just 'what does that mean?'

Interviewer: Were you able to figure it out?

Wendy: I was a little bit. I would constantly make mistakes and that would cause things to not pan out and I would get frustrated ... It was fun stuff when it worked out. It was like 'woah, I just changed this website to look the way I wanted it to look.' ... It was exciting having that ability but it was frustrating when I couldn't actually use it correctly.

Interviewer: Have you ever tried to figure anything else out with computers?

Wendy: Not really. I'm better than my dad with computers, but not by much. I know how to copy and paste and search for stuff in a web page. When it comes to more in-depth stuff, I'm completely lost and almost always mess something up.

Wendy appears to have drawn excitement from the design elements of HTML, but she also expresses an (understandable) frustration for when things don't work properly. Furthermore, this frustration appears to be a boundary of entry for Wendy as she positions herself as a novice, dismissing common tasks and reiterating a lack of confidence in her abilities to use "more in depth stuff."

As the interview turned to spreadsheets, Wendy's expressions became noticeably brighter:

Wendy: I love Excel! I use Excel regularly. It's my preferred method for organizing stuff.

Contrast this enthusiasm to Wendy's earlier declaration that she is "absolutely terrible with computers." After describing how she uses Excel in her day-to-day life, Wendy goes on to describe her experiences in the physics class:

\footnotetext{
${ }^{1}$ Some excerpts are edited, here, for clarity and brevity.
}

Wendy: I really like Excel spreadsheets. I find them to be really interesting and I like putting in formulas and I'm not an expert, but [in physics class,] it's a lot easier than doing all the calculations by hand. I like that a lot better. ...

Interviewer: What was that experience like?

Wendy: It was cool having that technology at hand to just go as long as I know how these formulas work and I can type it in to the cell and have it calculate every single thing and all the data I've put in and, um, as long as I do it right.

Although Wendy is still positioning herself as a novice, she expresses a sense of joy and enthusiasm when using spreadsheets, calling them "cool" and "interesting." Importantly, in contrast to Wendy's trepidation towards programming, she finds spreadsheets sufficiently accessible that she frequently uses them outside of class.

\section{B. Results Across Participants}

Let us now turn to our other participants' affective stance towards both programming and spreadsheets. For the sake of brevity, we will focus on our participants' initial responses to being asked about programming and spreadsheets as these encapsulate the attitudes expressed throughout the interviews.

\section{1. "What do you think of when you think of programming?"}

When first asked about programming, most of our participants expressed a general sense of apprehension:

Trudy: [It's] hard. I've never done any kind of programming, so I'm not sure I'm gonna get the questions you're gonna ask me. I'll do my best; I'm just completely inexperienced with it.

Linda: ... I've actually never done anything with programming so I'm a little nervous to see what you would like me to do. I read in [the informed consent document] that you want me to interpret a code ... I don't know how I would do that.

Sandra: I know nothing about computer programming, period.

Much of this anxiety appears to stem from our participants' limited exposure to programming. In particular, Linda's statement, "I've actually never done anything with programming so I'm a little nervous," suggests this connection. Encouragingly, Trudy, despite her trepidation, still manages to express a sense of determination. Only Reneé, who had had experience programming, expressed an initial positive attitude:

Reneé: When I think of programming, I think about web design mostly because I have experience with it ... it's really interesting to me and I would like to understand how to do some computer programing but I don't think I have the time for that. 


\section{2. "What do you think about spreadsheets?"}

When the interviews turned towards a discussion of spreadsheets, our participants expressed attitudes that ranged from openness to outright excitement (Wendy: "I love Excel!").

Reneé: [Using Excel is] fun because I'm the only member of my group who knows how to use formulas ... I've been using those skills throughout high school. Whenever I didn't have a calculator on hand it's easy to open up Excel and type it in as an equation.

Trudy: I've found Excel to be pretty user intuitive. Normally I'm not that great with technology, but it's pretty easy to get. It's really satisfying too. Instead of having to sit there and draw the graph yourself, it's really easy to enter all the data and it does it for you.

Not all of our participants readily bought into the use of spreadsheets; Sandra provided little detail about her attitudes while Linda revealed that she had found spreadsheets to be a challenging component of another class:

Linda: Yea, I've used Excel before... I used it when I briefly took... a business [calculus] class. ... they were like 'you're not going to be able to use a calculator,' and we would have to use Excel in all our calculations. I found pretty quickly that it was not for me.

Encouragingly, when discussing her use of Excel in her physics class, Linda conceded that she had learned some skills from her business calculus class and that using Excel to do calculations in physics "wasn't too bad."

\section{DISCUSSION}

This work sheds light on how life-science majors might view the introduction of computational modeling activities within the context of an introductory physics course and how we might need to scaffold these activities. In particular, our study suggests that spreadsheets could provide an accessible entryway to computational modeling. While most of our participants took apprehensive stances towards the idea of programming, we were surprised at the overly positive attitudes expressed towards spreadsheets. Most promisingly, our participants expressed the sense that spreadsheets are accessible and useful in class and in work. While our participants should not be taken to be representative of the broader population, their responses nonetheless help us to better understand issues of perception and accessibility. In particular, perceptions of gender norms surely colored our participants' responses (all of whom happened to be female), however these biases still exist in the classroom and do not negate our interpretation of the results.

Anecdotally, a few students enrolled in the first author's general-level physics course (at Elon University) saw a poster based on this research and spontaneously commented that our example of a spreadsheet-based computational model looked accessible while our example of a VPython model looked complicated. We take the opposite stance, but these comments support our findings and further suggest that we use spreadsheets as a path-of-least-resistance to help scaffold lifescience majors to more sophisticated (and easier!) methods of computational modeling.

This exploratory study was designed to help bring further research questions into focus that then might be targeted by larger, future studies. In particular, this study suggests the need to better understand how the broader population of IPLS students might interact with spreadsheets in a classroom environment and how this might influence their affective stances towards physics, modeling, and science. Future efforts should be dedicated to exploring how we might best scaffold more sophisticated computational modeling practices for IPLS students and to developing instructional resources.

\section{ACKNOWLEDGEMENTS}

We would like to thank Anna Lewis for her help with the study design and data analysis; Ayush Gupta, Gina Quan, and Vashti Sawtelle for their discussions on methodology; Eleanor Close for her comments on an early draft; and the NCSU PER group and the Elon Undergraduate Research program for facilitating our research.
[1] D. Meredith and E. F. Redish, Physics Today pp. 38 - 43 (2013).

[2] R. Hilborn and D. Meredith, eds., Conference on Introductory Physics for the Life Sciences Report (2015).

[3] R. W. Chabay and B. A. Sherwood, American Journal of Physics 76, 307 (2008).

[4] B. L. Sherin, A. A. diSessa, and D. Hammer, Interactive Learning Environments 3, 91 (1993).

[5] E. F. Redish and J. Wilson, American Journal of Physics 61, 222 (1993).

[6] S. A. Weatherford, Ph.D. thesis, North Carolina State University (2011).

[7] B. R. Lunk, Ph.D. thesis, North Carolina State University (2012).
[8] http://circle4.com/biophysics/.

[9] R. Pekrun, T. Goetz, W. Titz, and R. P. Perry, Educational Psychologist 37, 91 (2010).

[10] G. M. Quan, A. Gupta, and A. Elby, in 2015 ASEE Proceedings (2015).

[11] G. Quan and A. Gupta, personal correspondence (2014).

[12] M. Banerjee, M. Capozzoli, L. McSweeney, and D. Sinha, The Canadian Journal of Statistics 27, 3 (1999).

[13] V. K. Otero and D. B. Harlow, Getting started in qualitative physics education research, American Association of Physics Teachers, Reviews in PER Vol. 2 (2009). 